\title{
Alterações clínicas e laboratoriais em equinos adaptados a dieta com nível elevado de concentrado e submetidos à sobrecarga dietética com amido
}

\section{Clinical and laboratorial changes in horses adapted to diet with high levels of concentrated and submitted to dietetic starch overload}

\author{
Tiago Marques dos SANTOS ${ }^{1}$; Fernando Queiroz de ALMEIDA²; Marcus Sandes PIRES ${ }^{1}$; Juliano \\ Martins SANTIAGO ${ }^{3}$; Erica Cristina Rocha ROIER ${ }^{1}$; Daniel Silva GUEDES JÚNIOR ${ }^{1}$; Yzabella Alves \\ Campos NOGUEIRA ${ }^{4}$; Lucas Santiago BRASILEIRO ${ }^{4}$
}

${ }^{1}$ Discente do Curso de Pós-graduação em Ciências Veterinárias do Instituto de Veterinária da Universidade Federal Rural do Rio de Janeiro, Seropédica-RJ, Brasil.

${ }_{2}^{2}$ Departamento de Medicina e Cirurgia Veterinária do Instituto de Veterinária da Universidade Federal Rural do Rio de Janeiro, Seropédica-RJ, Brasil

${ }^{3}$ Discente do Curso de Pós-graduação em Zootecnia do Instituto de Zootecnia da Universidade Federal Rural do Rio de Janeiro, Seropédica-RJ, Brasil

${ }^{4}$ Discente de Medicina Veterinária do Instituto de Veterinária da Universidade Federal Rural do Rio de Janeiro, Seropédica-RJ, Brasil

\begin{abstract}
Resumo
Este trabalho teve como objetivo avaliar o efeito da sobrecarga dietética com amido em equinos adaptados a dieta com nível elevado de concentrado. Seis equinos adultos foram previamente adaptados à dieta composta por feno de coast cross e concentrado na proporcão de 60:40 e, posteriormente submetidos à infusão gástrica de 17,6 g amido/kg de peso corporal. Os equinos foram avaliados por meio de exames clínicos e laboratoriais até 36 horas após a sobrecarga. Um equino veio a obito 32 horas após a sobrecarga, apresentando quadro clínico de endotoxemia e laminite. Não houve diferença $(\mathrm{P}>0,05)$ nas frequências cardíaca e respiratória, temperatura retal, tempo de preenchimento capilar e temperatura superficial dos cascos dos equinos durante o experimento. Houve tendência ao aumento na motilidade intestinal, seguido de aumento na fluidez das fezes. O volume globular e a concentracão de proteína plasmática total aumentaram ( $\mathrm{P}<0,05) 24$ horas após a sobrecarga quando comparados aos valores basais, de 26,7 a $32,0 \%$ e de 7,1 e $8,1 \mathrm{~g} / \mathrm{dL}$, respectivamente. Os neutrófilos bastonetes aumentaram $(\mathrm{P}<0,05)$ durante o período de avaliação, com valores variando de 0,1 a $0,7 \times 10^{3} / \mu \mathrm{L}$ antes da sobrecarga e 24 horas após, respectivamente, enquanto os eosinófilos apresentaram redução. A concentração plasmática de lactato teve aumento discreto com diferença $(\mathrm{P}<0,05)$ antes, $20 \mathrm{e}$ 28 horas após a sobrecarga, com valores de $0,7,1,0$ e 1,2 mmol/L, respectivamente. Conclui-se que equinos consumindo dieta com elevado nível de concentrado e submetidos à sobrecarga dietética com amido apresentam alterações clínicas e laboratoriais moderadas, porém, casos graves, seguidos do óbito, podem ocorrer devido à variação individual.
\end{abstract}

Palavras-chave: Equinos. Carboidratos. Sobrecarga. Clínica. Patologia Clínica.

\begin{abstract}
This work was carried out to evaluate the effect of dietetic starch overload in horses adapted to diets with high level of concentrated. Six crossbreed mature horses were previously adapted to diet composed by coast cross hay and concentrate in a $60: 40$ proportion and later submitted to gastric infusion of $17.6 \mathrm{~g}$ starch $/ \mathrm{kg}$ body weight. The horses were evaluated by clinical and laboratorial examination until 36-hour after overload. One horse died 32 hours after overload, presenting clinical signs of endotoxemia and laminitis. No differences $(P>0.05)$ were observed in heart and respiratory rate, rectal temperature, capillary refill time and hoof temperature of horses. There was a tendency to increase intestinal mobility following by fluidity of faeces. Increase $(\mathrm{P}<0.05)$ in packed cell volume and total plasma protein were observed 24 hours after overload when compared to baseline, from 26.7 to $32.0 \%$ and 7.1 to $8.1 \mathrm{~g} / \mathrm{dL}$, respectively. Neutrophils increased $\left(\mathrm{P}<0.05\right.$ ) during the evaluation period, with values varying of 0.1 to $0.7 \times 10^{3} / \mu \mathrm{L}$ before overload and 24 hours after, respectively, while the eosinophils decreased. The plasma lactate concentration was slight increased with difference $(\mathrm{p}<0.05)$ before, 20 and 28 hours after overload, with values of $0.7,1.0$ and $1.2 \mathrm{mmol} / \mathrm{L}$, respectively. It was concluded that horses consuming diets with high levels of concentrated and submitted to dietetic starch overload had clinical and laboratory changes moderate, however, severe cases, followed by death may occur due to individual variation.
\end{abstract}

Keywords: Equine. Carbohydrate. Overload. Clinical. Clinical Pathology. 


\section{Introdução}

O abdômen agudo é uma importante causa de morbidade e mortalidade na população equina ${ }^{1}$. Estudos epidemiológicos apontam como fatores de risco práticas de alimentação utilizando dietas contendo elevadas quantidades de grãos, os tipos de volumoso utilizados, os tipos de grãos, menor exposição à pastagem e idade acima de oito ou dez anos ${ }^{2,3}$, além de outros fatores relacionados ao tempo de estabulação e alterações no treinamento ${ }^{4}$.

Em equinos alimentados com dietas com altos teores de amido, a capacidade de digestão do amido no intestino delgado pode ser ultrapassada e o amido ser fermentado no intestino grosso resultando em acidificação da digesta devido ao aumento na produção de ácidos graxos voláteis (AGVs) e ácido lático ${ }^{5}$. Segundo Potter et al. ${ }^{6}$, o limiar da digestão do amido no intestino delgado é de 3,5 a 4,0 g de amido/ $\mathrm{kg}$ de peso corporal (PC) em cada refeição. A digesta ácida no intestino grosso pode iniciar efeitos secundários como irritação e degeneração do epitélio intestinal ${ }^{7}$, além de promover a morte de bactérias gram-negativas e liberação de endotoxinas que, se absorvidas, produzem quadro clínico de endotoxemia ${ }^{8}$.

Vários estudos utilizaram a metodologia da sobrecarga dietética com amido como ferramenta de avaliação da patofisiologia da laminite em equinos ${ }^{8,9,10,11,12,13}$. Em síntese, os equinos apresentaram quadro clínico de cólica, aumento da concentração plasmática de lactato e do nível sérico de endotoxina antecedendo o quadro clínico de laminite, o qual variou de 32 a
48 horas após a sobrecarga. A importância do presente estudo quando comparado aos referidos anteriormente é o fato dos equinos utilizados aqui serem adaptados à dieta com nível elevado de concentrado, o que pode torná-los mais resistentes aos distúrbios gastrointestinais e sistêmicos em função da fermentação do amido no intestino grosso. Este trabalho teve como objetivo avaliar, no período de 36 horas, as alterações clínicas, hematológicas e bioquímicas em equinos alimentados com nível elevado de concentrado e submetidos à sobrecarga dietética com amido.

\section{Material e Método}

Foram utilizados seis equinos adultos, sem raça definida, com peso corporal variando de 335 a $393 \mathrm{~kg}$, machos, castrados e com idade variando de 17 a 27 anos. Os animais foram mantidos em baias individuais de $6 \mathrm{~m}^{2}$, e adaptados à dieta composta por feno de coast cross e concentrado, na proporção de 60:40 (Tabela 1), durante 15 dias. O projeto foi aprovado pela Comissão de Ética do Instituto de Veterinária da UFRRJ: 23083.011750/2006-33.

A sobrecarga dietética com amido foi realizada, com os equinos em jejum hídrico de 12 horas, via sonda nasogástrica, utilizando amido de milho (Maizena Duryea $^{\circledR}$ ) na proporção de $17,6 \mathrm{~g}$ de amido/kg de $\mathrm{PC}^{9}$ diluído em água na proporção de $757 \mathrm{~g}$ de amido por $1.000 \mathrm{~mL}$ de água. A infusão gástrica ocorreu durante 60 minutos, em três etapas, com a administração de $1 / 3$ do amido diluído em água por etapa. O período experimental correspondeu a 36 horas. Durante esse

Tabela 1 - Composição bromatológica, na base da matéria seca, do feno de coast cross e concentrado comercial utilizados na formulação da dieta para adaptação dos equinos

\begin{tabular}{cccccccccc}
\hline Alimento & $\begin{array}{c}\text { MS } \\
(\%)\end{array}$ & $\begin{array}{c}\text { MO } \\
(\%)\end{array}$ & $\begin{array}{c}\text { PB } \\
(\%)\end{array}$ & $\begin{array}{c}\mathrm{EE} \\
(\%)\end{array}$ & $\begin{array}{c}\mathrm{FDN} \\
(\%)\end{array}$ & $\begin{array}{c}\mathrm{FDA} \\
(\%)\end{array}$ & $\begin{array}{c}\mathrm{LIG} \\
(\%)\end{array}$ & $\begin{array}{c}\mathrm{EB} \\
\mathrm{Mcal} / \mathrm{Kg}\end{array}$ & $\begin{array}{c}\mathrm{CHO}_{\mathrm{H}} \\
(\%)\end{array}$ \\
\hline Feno de Coast cross & 88,4 & 93,8 & 11,9 & 1,26 & 67,21 & 31,44 & 2,43 & 3,49 & 1,96 \\
Ração Comercial & 88,7 & 82,8 & 11,1 & 3,73 & 44,77 & 18,44 & 1,57 & 3,45 & 37,34 \\
\hline
\end{tabular}

MS: matéria seca; MO: matéria orgânica; PB: proteína bruta; FDN: fibra em detergente neutro; FDA: fibra em detergente ácido; LIG: lignina; EB: energia bruta; $\mathrm{CHO}_{\mathrm{H}}$ : carboidrato hidrolisável. 
período os equinos tiveram acesso ad libitum a água, enquanto que o alimento concentrado e o volumoso foram oferecidos aos animais apenas para avaliação da manifestação de apetite.

Os exames clínicos foram procedidos, nos seis equinos, antes e a cada quatro horas até 24 horas após a sobrecarga dietética com amido. Posteriormente, três equinos prosseguiram na avaliação até o fim do período experimental e os demais foram eutanasiados para avaliação histopatológica e imuno-histoquímica da mucosa do trato gastrointestinal. Avaliou-se a manifestação de apetite, as frequências cardíaca e respiratória, motilidade intestinal, temperatura retal, tempo de preenchimento capilar (TPC), aspecto das mucosas oral e ocular, aspecto das fezes, comportamento do animal, pulso da artéria digital superficial (PADS) e a temperatura superficial dos cascos (TSC) através de termômetro de infravermelho (MiniTemp MT4, Raytek). Para quantificação da concentração plasmática de lactato e glicose, amostras de sangue foram coletadas, nos horários descritos anteriormente, por venopunção da jugular em tubos a vácuo contendo fluoreto de sódio. Após centrifugação, o plasma foi extraído e acondicionado em microtubos de polipropileno de $1,5 \mathrm{~mL}$ identificados e armazenados à $-20^{\circ} \mathrm{C}$ para análises com kits comerciais (Katal e Bioclin). As análises foram realizadas no período máximo de 24 horas após a coleta.

Para as outras avaliações laboratoriais e a quantificação de endotoxinas séricas, as amostras de sangue foram coletadas antes, 12, 24 e 36 horas após a sobrecarga por venopunção jugular, em tubos a vácuo contendo ácido etilenodiamino tetra-acético (EDTA) e imediatamente submetidas à realização do hemograma ${ }^{14}$, dosagem de fibrinogênio e proteína plasmática total (PPT), por refratometria. Para quantificação de endotoxinas, amostras com cerca de $9 \mathrm{~mL}$ de sangue foram coletadas em tubos a vácuo sem anticoagulante, centrifugadas, e o soro acondicionado em microtubos de polipropileno de 1,5 mL identificados e armazenados à $-20{ }^{\circ} \mathrm{C}$ para análise através do sistema Limulus Amebocyte Lysate (LAL) cromogênico quantitativo, com kit comercial (QCL$1000^{\circ}$, Cambrex ) e dosagem através de espectrofotometria (Biotek Reader).

Os resultados obtidos foram submetidos à análise de variância e comparados pelo teste t de Student, em nível de $5 \%$ de significância utilizando o programa Sistema de Análises Estatísticas e Genéticas - SAEG $8.0^{15}$.

\section{Resultados e Discussão}

Dos seis equinos submetidos à sobrecarga, cinco manifestaram apetite, mesmo que reduzido, durante o período de observação. No entanto, um equino apresentou inapetência quatro horas após a sobrecarga permanecendo inapetente até 32 horas, momento que veio a óbito. As alterações clínicas e laboratoriais apresentadas por este animal tornaram-se evidentes oito horas após a sobrecarga, seguida de agravamento do quadro clínico até o óbito: frequência cardíaca de 108 bpm, temperatura retal de 37,1 a $39,6^{\circ} \mathrm{C}$, TPC de 7 segundos, VG de 51\%, PPT de 10,0 g/dL e concentração plasmática de lactato de 4,3 mmol/L. Além dessas alterações, observou-se claudicação grau $\mathrm{II}^{16}$, congestão das mucosas ocular e oral e motilidade intestinal aumentada seguida de diarreia intensa. A concentração sérica de endotoxinas não foi quantificada neste animal, no entanto, os resultados clínicos e laboratoriais observados estão em acordo com os apresentados por equinos com quadro de endotoxemia e laminite ${ }^{9,10,13}$. Os resultados obtidos deste animal não foram incluídos nas análises estatísticas para comparação de médias, uma vez que superestima os valores médios apresentados pelos demais equinos. Isso demonstra a existência de variação individual entre equinos adaptados a uma alimentação com nível elevado de concentrado no que se refere ao risco de desenvolvimento de endotoxemia e laminite, quando submetidos à sobrecarga com amido. 
Entre os cinco equinos, um animal apresentou aumento no PADS dos membros posteriores 20 horas após a sobrecarga, evoluindo para claudicação grau $\mathrm{I}^{16}$ no membro posterior esquerdo. Não houve diferença $(p>0,05)$ na TSC com valores variando de 30,0 a $32,2{ }^{\circ} \mathrm{C}$. Pollitt e Davies ${ }^{11}$ observaram temperatura dos cascos próxima à temperatura ambiente, de $13,6^{\circ} \mathrm{C}$, seguido de elevação entre quatro e 15 horas após a sobrecarga. Nos equinos com laminite, a TSC atingiu $24,9^{\circ} \mathrm{C}$ às 24 horas da sobrecarga, superior aos animais controle, de $16,4^{\circ} \mathrm{C}$. A temperatura ambiente na qual foi realizado o estudo foi em torno de $10^{\circ} \mathrm{C}$, justificando os valores bem abaixo dos observados no presente estudo, com temperatura ambiente variando de 21,6 a $31,2^{\circ} \mathrm{C}$. Portanto, na avaliação da TSC deve-se considerar a temperatura ambiente em que o animal se encontra.

Houve alteração na motilidade intestinal em todos os equinos, sendo uns com aumento, seguido de quadros de fluidificação das fezes e/ou diarreia e, outros com redução da motilidade intestinal. No entanto, houve uma tendência de aumento na motilidade intestinal em função do tempo pós-sobrecarga. Essas alterações clínicas corroboram as descritas na literatura $^{13}$. A ocorrência de diarreia seguida de dor abdominal intermitente, de moderada a intensa também foi observada em outro experimento ${ }^{11}$. No presente estudo, os equinos não desenvolveram dor abdominal. Uma justificativa pode ser o aumento da flatulência apresentada cerca de 20 horas após a sobrecarga que, associada à motilidade intestinal aumentada, não propiciou acúmulo de gases no intestino grosso.

Os valores de frequência cardíaca e respiratória, temperatura retal e TPC não diferiram em função do tempo pós-sobrecarga (Tabela 2), não corroborando os resultados citados na literatura ${ }^{9,11,13}$. Em ambos os estudos, a frequência cardíaca apresentou valores acima de 70 bpm a partir de 36 horas após a sobrecarga, justificado, possivelmente pela dor e/ ou insuficiência cardiovascular decorrente da hemoconcentração e da disfunção circulatória. Estes valores estão acima dos observados no presente estudo, o que pode ser justificado pela adaptação dos equinos à dieta com nível elevado de concentrado e pelo fato de o período de avaliação deste estudo se restringir a 36 horas após a sobrecarga.

Os valores médios e desvio padrão do hemograma, PPT e fibrinogênio de equinos submetidos à sobrecarga dietética com amido estão citados na tabela 3. Observou-se aumento significativo $(\mathrm{P}<0,05)$ no VG e PPT, 24 horas após a sobrecarga. Antes da sobrecarga o VG apresentou valor médio de $26,7 \%$ seguido de aumento até 24 horas após a sobrecarga, permanecendo constante até 36 horas, com 32,7 e 32,0\%, respectivamente. Resposta semelhante também foi observada com os valores de PPT, os quais apresentaram aumento desde os valores basais até 36 horas após a sobrecarga, de 7,1 e 8,7 g/dL, respectivamente. $\mathrm{O}$ aumento não significativo observado no número de hemácias e de hemoglobina associado ao aumen-

Tabela 2 - Média e desvio padrão da frequência cardíaca, frequência respiratória, temperatura retal e tempo de preenchimento capilar em equinos submetidos à sobrecarga dietética com amido

\begin{tabular}{cccccc}
\hline $\begin{array}{c}\text { Parâmetros } \\
\text { Clínicos }\end{array}$ & \multicolumn{4}{c}{ Tempo após a sobrecarga dietética com amido (horas) } \\
\cline { 2 - 5 }$\left(\begin{array}{c}12 \\
(\mathrm{n}=5)\end{array}\right.$ & $\begin{array}{c}24 \\
(\mathrm{n}=5)\end{array}$ & $\begin{array}{c}36 \\
(\mathrm{n}=2)\end{array}$ \\
\hline Frequência cardíaca $(\mathrm{bpm})$ & $36,0 \pm 2,5^{\mathrm{a}}$ & $40,0 \pm 3,6^{\mathrm{a}}$ & $40,8 \pm 7,8^{\mathrm{a}}$ & $38,0 \pm 8,5^{\mathrm{a}}$ & 0,478 \\
Frequência respiratória $(\mathrm{mpm})$ & $18,0 \pm 2,2^{\mathrm{a}}$ & $20,0 \pm 3,6^{\mathrm{a}}$ & $22,0 \pm 7,5^{\mathrm{a}}$ & $14,0 \pm 2,8^{\mathrm{a}}$ & 0,057 \\
Temperatura retal $\left({ }^{\circ} \mathrm{C}\right)$ & $37,5 \pm 0,2^{\mathrm{a}}$ & $37,5 \pm 0,4^{\mathrm{a}}$ & $37,7 \pm 0,5^{\mathrm{a}}$ & $37,3 \pm 0,9^{\mathrm{a}}$ & 0,697 \\
Tempo de preenchimento capilar (segundos) & $2,0 \pm 0,0^{\mathrm{a}}$ & $2,0 \pm 0,0^{\mathrm{a}}$ & $2,67 \pm 0,5^{\mathrm{a}}$ & $2,5 \pm 0,7^{\mathrm{a}}$ & 0,362 \\
\hline
\end{tabular}

${ }^{\mathrm{a}}$ Médias, nas linhas, seguidas de letras iguais não diferem entre si pelo teste $t$ de Student $(\mathrm{P}>0,05)$. 
Tabela 3 - Média e desvio padrão do hemograma, proteína plasmática total e fibrinogênio de equinos submetidos à sobrecarga dietética com amido

\begin{tabular}{|c|c|c|c|c|c|}
\hline \multirow{2}{*}{ Parâmetros } & \multicolumn{4}{|c|}{ Tempo após a sobrecarga dietética com amido (horas) } & \multirow{2}{*}{ Valor de $P$} \\
\hline & $(\mathrm{n} \stackrel{0}{=} 5)$ & $\begin{array}{c}12 \\
(n=5)\end{array}$ & $\begin{array}{c}24 \\
(\mathrm{n}=5)\end{array}$ & $\begin{array}{c}36 \\
(n=2)\end{array}$ & \\
\hline VG (\%) & $26,7 \pm 2,2^{b}$ & $27,3 \pm 1,6^{b}$ & $32,7 \pm 4,6^{\mathrm{a}}$ & $32,0 \pm 5,6^{\mathrm{a}}$ & 0,025 \\
\hline $\mathrm{He}\left(\mathrm{x} 10^{6} / \mathrm{L}\right)$ & $4,9 \pm 0,5^{\mathrm{a}}$ & $5,1 \pm 0,7^{\mathrm{a}}$ & $5,27 \pm 0,5^{\mathrm{a}}$ & $5,4 \pm 8,8^{a}$ & 0,380 \\
\hline $\mathrm{Hb}(\mathrm{g} / \mathrm{dL})$ & $8,9 \pm 0,6^{\mathrm{a}}$ & $8,6 \pm 0,8^{a}$ & $9,9 \pm 1,4^{\mathrm{a}}$ & $9,3 \pm 0,1^{\mathrm{a}}$ & 0,167 \\
\hline VCM (fl) & $54,8 \pm 4,7^{\mathrm{a}}$ & $54,8 \pm 5,8^{\mathrm{a}}$ & $62,6 \pm 11,8^{\mathrm{a}}$ & $60,1 \pm 1,1^{\mathrm{a}}$ & 0,075 \\
\hline CHCM (g/dL) & $33,7 \pm 2,1^{\mathrm{a}}$ & $31,6 \pm 3,6^{\mathrm{a}}$ & $30,8 \pm 5,4^{\mathrm{a}}$ & $29,9 \pm 12,9^{a}$ & 0,079 \\
\hline Leucócitos $\left(\mathrm{x} 10^{3} / \mu \mathrm{L}\right)$ & $9,6 \pm 1,5^{\mathrm{a}}$ & $8,9 \pm 4,9^{a}$ & $13,7 \pm 3,6^{\mathrm{a}}$ & $9,8 \pm 2,5^{\mathrm{a}}$ & 0,188 \\
\hline Linfócitos $\left(\mathrm{x} 10^{3} / \mu \mathrm{L}\right)$ & $3,8 \pm 0,9^{a}$ & $3,3 \pm 2,1^{\mathrm{a}}$ & $4,8 \pm 1,4^{\mathrm{a}}$ & $3,9 \pm 1,7^{\mathrm{a}}$ & 0,401 \\
\hline Eosinófilos $\left(\mathrm{x} 10^{3} / \mu \mathrm{L}\right)$ & $0,4 \pm 0,1^{\mathrm{a}}$ & $0,2 \pm 0,2^{\mathrm{a}}$ & $0,3 \pm 0,1^{\mathrm{a}}$ & $0,1 \pm 0,8^{\mathrm{a}}$ & 0,051 \\
\hline Monócitos $\left(\mathrm{x} 10^{3} / \mu \mathrm{L}\right)$ & $0,4 \pm 0,1^{\mathrm{a}}$ & $0,4 \pm 0,2^{\mathrm{a}}$ & $0,5 \pm 0,3^{\mathrm{a}}$ & $0,4 \pm 2,5^{\mathrm{a}}$ & 0,452 \\
\hline Neutrófilos $\left(\mathrm{x} 10^{3} / \mu \mathrm{L}\right)$ & $4,9 \pm 1,1^{\mathrm{a}}$ & $4,7 \pm 2,7^{a}$ & $7,4 \pm 2,9^{a}$ & $4,8 \pm 1,5^{\mathrm{a}}$ & 0,246 \\
\hline Bastonetes $\left(\mathrm{x} 10^{3} / \mu \mathrm{L}\right)$ & $0,1 \pm 0,1^{b}$ & $0,2 \pm 0,2^{b}$ & $0,7 \pm 0,7^{a}$ & $0,5 \pm 0,2^{\mathrm{a}}$ & 0,044 \\
\hline PPT (g/dL) & $7,1 \pm 0,7^{d}$ & $7,5 \pm 1,1^{\mathrm{c}}$ & $8,1 \pm 1,3^{b}$ & $8,7 \pm 0,1^{\mathrm{a}}$ & 0,002 \\
\hline FIB (mg/dL) & $433,3 \pm 225,1^{\mathrm{a}}$ & $433,3 \pm 196,6^{a}$ & $500,0 \pm 244,9^{\mathrm{a}}$ & $700,0 \pm 141,4^{\mathrm{a}}$ & 0,146 \\
\hline
\end{tabular}

${ }^{a}$ Médias, nas linhas, seguidas de letras iguais não diferem entre si pelo teste $t$ de Student $(P>0,05)$.

VG: volume globular; He: hemácias; Hb: hemoglobina; VCM: volume globular médio; CHCM: concentração de hemoglobina globular médio; PPT: proteína plasmática total; FIB: fibrinogênio.

to $(\mathrm{P}<0,05)$ no VG e PPT pode ser justificado pela hemoconcentração ocasionada pela desidratação. Esse quadro está associado ao aumento da produção de AGVs e ácido lático no intestino grosso, os quais promovem aumento na pressão osmótica do lúmen intestinal resultando em fluxo de água da corrente sanguínea para o intestino ${ }^{17}$. Além disso, a hemoconcentração pode ser justificada pelo consumo reduzido de água durante o período experimental. Mesmo não havendo diferença $(\mathrm{p}>0,05)$, houve aumento nos valores de fibrinogênio a partir de 24 horas após a sobrecarga.

$\mathrm{Na}$ avaliação do leucograma, apenas os bastonetes apresentaram diferença $(\mathrm{p}<0,05)$ durante o período de avaliação, com valores variando de 0,1 a $0,7 \times 10^{3}$ / $\mu \mathrm{L}$ antes da sobrecarga e 24 horas após, respectivamente, seguidos por uma discreta redução às 36 horas, de $0,5 \times 10^{3} / \mu \mathrm{L}$. A redução não significativa observada no número de eosinófilos e aumento dos neutrófilos se justifica pelo estresse sistêmico ${ }^{18}$. Os resultados do hemograma, PPT e fibrinogênio observados estão concordantes com os citados na literatura corrente ${ }^{9,13}$. As alterações no leucograma observadas no presente estudo corroboram, em parte, com as observadas por Martins Filho et al. ${ }^{13}$.Esses autores observaram aumento discreto não significativo, porém, progressivo no número de leucócitos nos animais submetidos à sobrecarga. No entanto, no presente estudo o aumento não significativo foi observado somente 24 horas após a sobrecarga, seguido de redução ao final do período experimental. A literatura ${ }^{19,20}$ relata leucocitose bem definida 28 e 32 horas após a sobrecarga, no entanto, os equinos não eram adaptados à dieta com elevado nível de concentrado. A adaptação ao consumo de concentrado e a hemoconcentração observada poderia justificar o aumento discreto no número de leucócitos no presente estudo.

A concentração plasmática de lactato (Tabela 4) apresentou tendência de aumento em função da sobrecarga dietética com amido, com valores variando de 0,7 a 1,2 $\mathrm{mmol} / \mathrm{L}$. Até oito horas após a sobrecarga, a concentração de lactato apresentou discreto aumento, diferindo $(\mathrm{P}<0,05)$ dos níveis basais, de 0,7 e $1,0 \mathrm{mmol} / \mathrm{L}$, respectivamente. Além disso, houve diferença $(\mathrm{P}<0,05)$ na concentração plasmática de lactato as 20 e 28 horas 
Tabela 4 - Média e desvio padrão da concentração plasmática de lactato e glicose de equinos submetidos à sobrecarga dietética com amido

\begin{tabular}{|c|c|c|c|c|c|c|c|c|c|c|c|}
\hline \multirow{2}{*}{ Parâmetros } & \multicolumn{10}{|c|}{ Tempo após a sobrecarga dietética com amido (horas) } & \multirow[b]{2}{*}{ Valor de $P$} \\
\hline & $(\mathrm{n}=5)$ & $(n \stackrel{4}{=} 5)$ & $(\mathrm{n}=5)$ & $(\mathrm{n}=5)$ & $(\mathrm{n}=5)$ & $\begin{array}{c}20 \\
(n=5)\end{array}$ & $\begin{array}{c}24 \\
(\mathrm{n}=5)\end{array}$ & $\begin{array}{c}28 \\
(n=2)\end{array}$ & $\begin{array}{c}32 \\
(n=2)\end{array}$ & $\begin{array}{c}36 \\
(n=2)\end{array}$ & \\
\hline $\begin{array}{c}\text { Lactato } \\
(\mathrm{mmol} / \mathrm{L})\end{array}$ & $0,7 \pm 0,2^{\mathrm{d}}$ & $0,9 \pm 0,1^{\mathrm{cd}}$ & $1,0 \pm 0,3^{\mathrm{bc}}$ & $0,7 \pm 0,1^{\mathrm{d}}$ & $0,9 \pm 0,2^{\mathrm{bc}}$ & $1,0 \pm 0,3^{\mathrm{b}}$ & $0,9 \pm 0,3^{\mathrm{bcd}}$ & $1,2 \pm 0,3^{\mathrm{a}}$ & $0,9 \pm 0,3^{\text {bcd }}$ & $1,0 \pm 0,3^{\mathrm{bc}}$ & 0,011 \\
\hline $\begin{array}{l}\text { Glicose } \\
(\mathrm{mg} / \mathrm{dL})\end{array}$ & $78,3 \pm 0,6^{\mathrm{d}}$ & $118,7 \pm 45,4^{\text {ab }}$ & $92,5 \pm 14,2^{\text {cd }}$ & $87,3 \pm 12,9^{\mathrm{cd}}$ & $80,6 \pm 12,5^{\mathrm{cd}}$ & $86,6 \pm 9,9^{\mathrm{cd}}$ & $89,6 \pm 7,0^{\text {cd }}$ & $101,7 \pm 9,1^{\mathrm{bc}}$ & $85,0 \pm 17,0^{\text {cd }}$ & $84,0 \pm 8,5^{\mathrm{cd}}$ & 0,002 \\
\hline
\end{tabular}

${ }^{a}$ Médias, nas linhas, seguidas letras iguais não diferem entre si pelo teste $t$ de Student $(P>0,05)$.

após a sobrecarga quando comparado aos níveis basais, com valores de 1,0, 1,2 e 0,7 mmol/L, respectivamente.

$\mathrm{O}$ aumento na concentração plasmática de lactato observado no presente estudo pode ser explicado pelo excesso de carboidratos hidrolisáveis disponibilizados para fermentação microbiana no ceco-cólon resultando em produção excessiva de AGVs e ácido lático ${ }^{21}$. Isso se justifica pelos valores de $\mathrm{pH}$ do cólon abaixo de 6,022, além da redução do pH $(6,09$ a 4,46) e da capacidade de tamponamento das fezes de equinos em função do tempo após a sobrecarga ${ }^{23}$. Por outro lado, a pequena variação na concentração de lactato pode ser justificada pelo fato dos equinos estarem adaptados a dietas ricas em concentrado e, desta forma, já existirem bactérias capazes de metabolizar o ácido lático em AGVs, impedindo seu acúmulo no intestino grosso ${ }^{24}$. Além disso, o quadro de hemoconcentração pode contribuir para este aumento, uma vez que há perda de água para o lúmen intestinal.

A concentração plasmática de glicose aumentou ( $\mathrm{P}<0,05)$ de forma súbita quatro horas após a sobrecarga, 118,7 mg/dL (Tabela 4). Posteriormente, houve redução oito horas pós-sobrecarga permanecendo constante até 36 horas, com valores de 92,5 e 84,0 mg/ $\mathrm{dL}$, respectivamente. $\mathrm{O}$ aumento observado nos níveis de glicose, provavelmente é resultado da digestão do amido no intestino delgado. Não houve hiperglicemia com valores superiores a $250 \mathrm{mg} / \mathrm{dL}$, nem hiperglicemia transitória seguida por marcante hipoglicemia observada em casos de endotoxemia ${ }^{24}$. Dessa forma, a curva glicêmica nos equinos do presente estudo apresentou um comportamento pós-prandial normal.
A concentração sérica de endotoxinas não ultrapassou 0,1000 EU/mL, limiar de sensibilidade do ensaio, podendo até mesmo estar ausente até 36 horas após a sobrecarga. Níveis séricos de endotoxinas inferiores a $0,1000 \mathrm{EU} / \mathrm{mL}$ associados à ausência de sinais clínicos de endotoxemia, juntamente com a discreta elevação na concentração plasmática de lactato e níveis plasmáticos de glicose normais, sugerem que até 36 horas após a sobrecarga não houve lesão suficiente na mucosa do trato gastrointestinal para que os animais desenvolvessem quadro clínico grave de endotoxemia e acidose metabólica e posteriormente laminite $e^{9,11,25}$.

\section{Conclusões}

Equinos adaptados à dieta com elevado nível de concentrado e submetidos à sobrecarga dietética com amido apresentam alterações clínicas moderadas até 36 horas pós-sobrecarga, porém, alterações graves, seguida do óbito, podem ocorrer em função da variação individual. Além disso, a sobrecarga dietética com amido utilizado no presente estudo promove uma fermentação intensa do amido no intestino grosso causando aumento discreto na concentração plasmática de lactato sem acarretar quadro clínico de endotoxemia, 36 horas pós-sobrecarga. Isso demonstra que equinos consumindo dieta com elevado nível de concentrado e submetidos à sobrecarga dietética com amido são menos susceptíveis à apresentação de distúrbios gastrointestinais e sistêmicos em função da fermentação do amido no intestino grosso. 


\section{Referências}

1. SOUTHWOOD, L. L. Acute abdomen. Clinical Techniques in Equine Practice, v. 5, n. 2, p. 112-126, 2006.

2. TINKER, M. K.; WHITE, N. A.; LESSARD, P.; THATCHER, C. D.; PELZER, K. D.; DAVIS, B.; CARMEL, D. K. Prospective study of equine colic risk factors. Equine Veterinary Journal, v. 29, n. 6 , p. $454-458,1997$.

3. HUDSON, J. M.; COHEN, N. D.; GIBBS, P. G.; THOMPSON, J. A. Feeding practices associated with colic in horses. Journal of the American Veterinary Medical Association, v. 219, p. 1419-1425, 2001.

4. HILlYER, M. H.; TAYLOR, F. G. R.; PROUDMAN, C. J.; EDWARDS, G. B.; SMITH, J. E.; FRENCH, N. P. Case control study to identify risk factors for simple colonic obstruction and distention colic in horses. Equine Veterinary Journal, v. 34, n. 5, p. 455-463, 2002.

5.ZEYNER, A.; GEIBLER, C.; DITTRICH, A. Effects of hay intake and feeding sequence on variables in faeces and faecal water (dry matter, $\mathrm{pH}$ value, organic acids, ammonia, buffering capacity) of horses. Journal of Animal Physiology and Animal Nutrition, v. 88, n. 1, p. 7-19, 2004.

6. POTTER, G. D.; ARNOLD, F. F.; HOUSEHOLDER, D. D.; HANSEN, D. H.; BROWN, K. M. Digestion of starch in the small or large intestine of the equine. Pferdeheilkunde, v. $1, \mathrm{n}$. 4, p. 107-111, 1992.

7. KRUEGER, A. S.; KINDER, D. A.; GARNER, H. E.; SPROUSE, R. F. Ultrastructural study of the equine cecum during onset of laminitis. American Journal of Veterinary Research, v. 47, n. 8, p. 1804-1812, 1986

8. MOORE, J. N.; GARNER, H. E.; BERG, J. N.; SPROUSE, R. F. Intracecal endotoxin and lactate during the onset of equine laminitis: A preliminary report. American Journal of Veterinary Research, v. 40, n. 5, p. 722-723, 1979.

9. GARNER, H. E.; COFFMAN, J. R.; HAHN, A. W.; HUTCHESON, D. P.; TUMBLESON, M. E. Equine laminitis of alimentary origin: An experimental model. American Journal of Veterinary Research, v. 36, n. 4, p. 441-444, 1975.

10.SPROUSE, R. F.; GARNER, H. E.; GREEN, E. M. Plasma endotoxin levels in horses subjected to carbohydrate induced laminitis. Equine Veterinary Journal, v. 19, n. 1, p. 25-28, 1987.

11.POLLITT, C. C.; DAVIES, C. T. Equine laminitis: its development coincides with increased sublamellar blood flow. Equine Veterinary Journal, v. 26, p. 125-132, 1998.

12.PAES LEME, F. O.; WURZINGER, L. J.; VASCONCELOS, A. C.; ALVES, G. E. S. Ativação de plaquetas de equinos com laminite induzida e tratados com ketropofeno, fenilbutazona e flunixin meglumina. Arquivo Brasileiro de Medicina Veterinária e Zootecnia, v. 58, n. 2, p. 149-157, 2006.
13. MARTINS FILHO, L. P.; FAGLIARI, J. J.; MORAES, J. R. E.; SAMPAIO, R. C.; OLIVEIRA, J. A.; LACERDA NETO, J. C. Estudo clínico e laboratorial da fase prodômica da laminite equina induzida por sobrecarga de carboidrato. ARS Veterinária, v. 23, n. 1, p. 32-39, 2007.

14. COLES, E. H. (Ed.). Patologia clínica veterinária. São Paulo: Manole, 1984. 566 p.

15. UNIVERSIDADE FEDERAL DE VIÇOSA. SAEG. Sistema de análises estatísticas e genéticas. Viçosa: Universidade Federal de Viçosa, 2000. 150 p. Manual do usuário.

16. OBEL, N. Studies on the histopathology of acute laminitis. Veterinary Stockholm, v. 1, n. 1, p. 1-50, 1948.

17. ARGENZIO, R. A. Digestão e absorção dos carboidratos, gorduras e proteínas. In: DUKES, H. H. Fisiologia dos animais domésticos. 11. ed. Rio de Janeiro: Guanabara Koogan, 1996. p. 330-342.

18.HOOD, D. M.; AMOSS, M. S.; GREMMEL, S. M.; HIGHTOWER, D. Heparin as a preventative for equine laminitis. In: EQUINE ENDOTOXEMIA LAMINITIS SYMPOSIUM, 1., 1980, Lexington. Proceedings... Lexington, 1980. p. 146-149.

19. WEISS, D. J. Equine laminitis: a review of recent research. Equine Practice, v. 19, n. 1, p. 16-20, 1997.

20.WEISS, D. J.; TRENT, A. M.; JOHNSTON, G. Prothrombotic events in the prodromal stages of acute laminitis in horses. American Journal of Veterinary Research, v. 56, p. 986-991, 1995.

21.ROWE, J. B.; PETHICK, D. W.; JOHNSON, K. G. Controlling acidosis in the equine hindgut. In: ROWE, J. B.; NOLAN, J. V. Recent advances in animal nutrition in Australia. Australia: Department of Animal Science, University of New England, 1995. p. 136-142.

22.SANTOS, T. M. Efeitos gastrointestinais e sistêmicos em equinos submetidos à sobrecarga dietética com amido. 2007. 68 f. Dissertação (Mestrado em Ciências Veterinárias) Universidade Federal Rural do Rio de Janeiro, Seropédica, 2007.

23.SANTOS, T. M.; ALMEIDA, F. Q.; GODOI, F. N.; SILVA, V. P.; FRANÇA, A. B.; SANTIAGO, J. M.; SANTOS, C. S. Capacidade tamponante, $\mathrm{pH}$ e consistência das fezes em equinos submetidos à sobrecarga dietética com amido. Ciência Rural, v. 39, n. 6, p. 1782-1788, 2009.

24.SMITH, B. P. (Ed.). Tratado de medicina interna de grandes animais. São Paulo: Manole, 1993. 900 p.

25.KING, J. N.; GERRING, E. L. Detection of endotoxin in cases of equine colic. The Veterinary Record, v. 123, n. 10, p. 269-271, 1988. 